\title{
Improvement of Walking Support System for Visually Impaired Person
}

\author{
Katsunobu Hatano ${ }^{\mathrm{a}}$, Shota Watabe ${ }^{\mathrm{b}}$, Yutaka Tange ${ }^{\mathrm{a},{ }^{,}}$, Hideaki Katayama ${ }^{\mathrm{a}}$ \\ ${ }^{a}$ National Institute of Technology, Maizuru College, Shiroya 234, Maizuru and 625-8511, Japan \\ ${ }^{\mathrm{b}}$ Mitsubishi Electric Engineering Co., Ltd., Kudankita 1-13-5, Chiyoda and 102-0073, Japan \\ *Corresponding Author: tange @maizuru-ct.ac.jp
}

\begin{abstract}
In our laboratory, we have developed an obstacle detection system using the smartphone for a visually impaired person ${ }^{(1)}$. But it was the system that depended on the height information of smartphone. So this system lacked practicality. In this study, we installed an ultrasonic sensor and 6-axis sensor in the white walking stick, and built a system which doesn't depend on the height information of the smartphone. As a result, by using the fabricated system, we found it possible to detect the static obstacle such as walls, stairs and other obstacles which prevent visually impaired persons from walking. And we have improved the system so that it can be compatible with the touch-technic done by the visually impaired parson in walking.
\end{abstract}

Keywords: visually impaired person, obstacle detection, white walking stick.

\section{Introduction}

The information that a healthy person receives from the outside world through the organs of perception is mostly visual, and amounts to $83 \%$ of the whole information. Because visually impaired persons can't receive information from vision, they detect obstacles by using a white walking stick. But the white walking stick has a narrow detectable range, so accidents on a platform at a station and at the stairs are constant. In our laboratory, we built a system which, by combining the ultrasonic distance sensor and the 6-axis sensor (the acceleration sensor and the angular velocity sensor) in the smartphone, detects objects. But this method needed the information of height from the ground beforehand to calculate distance from the obstacle. Besides, the smartphone had to be raised above the obstacle. So it was not a practical system.
This time, we have aimed at building a system of the detection of obstacles using a smartphone attached to a white walking stick without recourse to height information.

\section{Previous study}

In our previous study, we detected obstacles using the smartphone and the ultrasonic distance sensor. As shown in Fig.1, we used the difference between the real distance measured with the ultrasonic sensor and the estimated distance measured with the inclination angle of the smartphone.

By using the height $h$ of the smartphone from the ground and the inclination angle $\theta$ measured with the sensor of the smartphone, the estimated distance is given by the following formula.

$$
k=\frac{h}{\sin \left(\frac{\pi}{2}-\theta\right)}=\frac{h}{\cos (\theta)}
$$

$\Delta d$ is defined by the following formula.

$$
\Delta d=|k-d|
$$

By using the difference between the real distance $d$ and the estimated distance $k$, and defining the proper threshold $\varepsilon$, this system made it possible to detect obstacles.

But there were the following problems.

1. It needed the height information of the smartphone in advance, so we had to set it every time.

2. As the smartphone is installed in the white walking stick, so it becomes heavy.

3. It can detect only the static obstacles, and can't detect the dynamic obstacles.

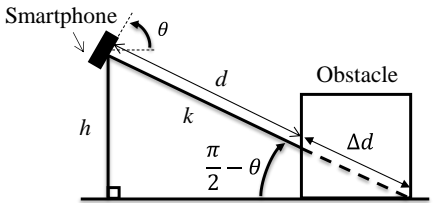

Fig.1 The detection principle in the previous study 


\section{Suggestion of new detection method}

\subsection{Big picture view of system}

Fig.2 gives the big picture view of the system of this study. In this study we aimed at building a system which detects not only static obstacles but also dynamic ones. Static obstacles are detected with the distance sensor and the 6-axis sensor attached to the white walking stick. The dynamic obstacles such as bicycles and cars are detected by the doppler sensor.

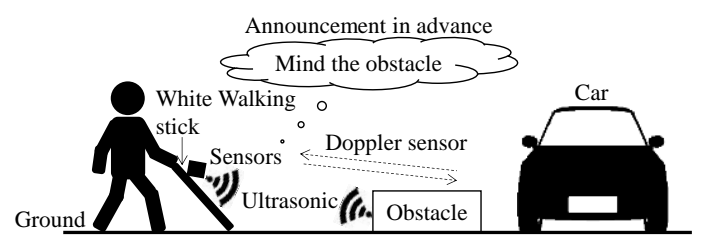

Fig.2 System diagram

\subsection{Detection method}

Fig. 3 gives the outline of the detection method. The new system detects obstacles by using the difference between the real distance $d$ measured with the ultrasonic sensor and the estimated distance $k$ measured on the basis of the inclination angle of the white walking stick.

The distance from the distance sensor to the end of the white walking stick is shown with $l$, the angle between the stick and the distance sensor is shown with $\phi$ (const), the angle between the stick and the ground is shown with $\theta$. By using trigonometric function, the relationship between the distance and the angle is shown with the following formula.

$$
\begin{gathered}
\cos (\theta+\varphi)=\frac{h}{k} \\
\cos \theta=\frac{h}{l}
\end{gathered}
$$

From the formula (3) and formula (4), the estimated distance $k$ is evaluated as formula (5).

$$
k=\ell \frac{\cos (\theta)}{\cos (\theta+\varphi)}
$$

The difference between the real distance $d$ and the estimated distance $k, \Delta d$ is defined by following formula.

$$
\Delta d=|k-d|
$$

The system can detect obstacles by defining the proper threshold $\varepsilon$ for the difference between the real distance $d$ and the estimated distance $k$ which is shown in formula (6) as $\Delta d$.

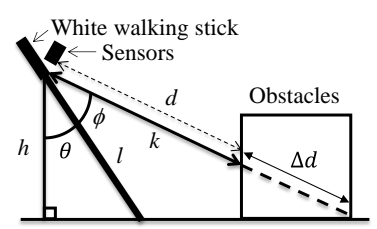

Fig.3 The method of detecting static obstacles

\section{Basic experiments and their results}

\subsection{Measurement of real distance and its result}

Fig. 4 shows the relationship between the real distance to the wall as an obstacle and the value calculated with the distance sensor. The calculation of distance to the obstacle is based on the time it takes for the ultrasonic wave reflected from the obstacle to return to the sensor. From the result, we have found that the distance can be detected up to $5.5 \mathrm{~m}$, and that its error rate is within $1.9 \%$.

Fig.5 shows the directivity distribution of the distance sensor. When the obstacle was placed on the points of $1 \mathrm{~m}$, $3 \mathrm{~m}$, and $5.5 \mathrm{~m}$, the sensor could detect it up to the angle of 25 degrees left and right from the center.

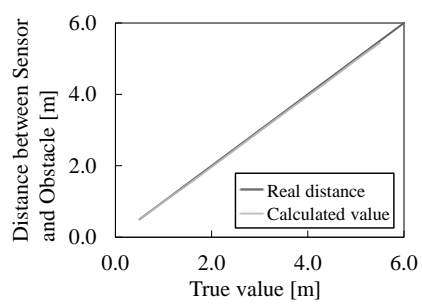

Fig.4 The relationship between the real distance and the calculated value

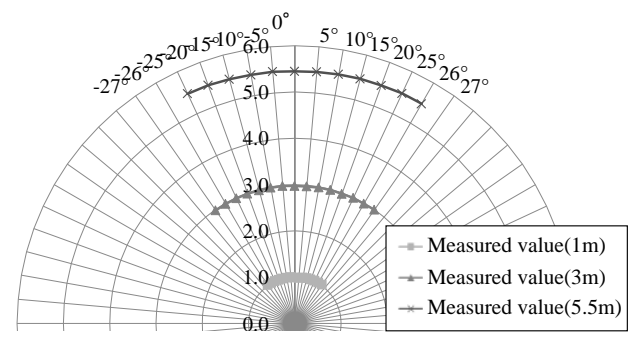

Fig.5 The directivity distribution of the distance sensor.

\subsection{Measurement of angle and its result}

Fig.6 shows the relationship between the true value measured with the measuring instrument (DML-280PRO, Digi-Pas) and the estimated value given by the angle sensor. The estimated value of the angle was calculated by using the 6-axis sensor (the angular velocity sensor and the acceleration sensor), and passing it through the Kalman filter. The error rate of the estimated value was within $3.4 \%$. 


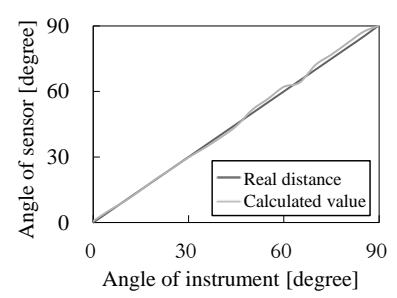

Fig.6 The relationship between the true value and the estimated value

\section{Evaluation experiment of detection ability}

\subsection{Method of experiment}

We have conducted a detection test, installing the system developed in this study to the white walking stick and supposing actual obstacles. This time, we had the system detect a wall, stairs and obstacle (height $35 \mathrm{~cm} \times$ width $35 \mathrm{~cm} \times$ depth $25 \mathrm{~cm}$ ). The test was done by the following process.

(1) As shown in Fig. 7, the tester stands $0.3 \mathrm{~m}$ away from the object of detection.

(2) The tester holds the white walking stick for $5 \mathrm{sec}$ and measures the real distance and the estimated distance.

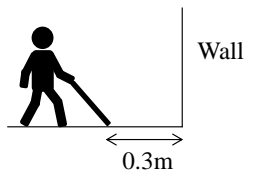

(a)Wall

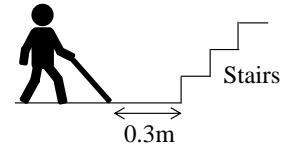

(c) Up-stair

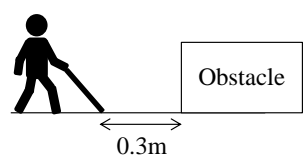

(b) Obstacle

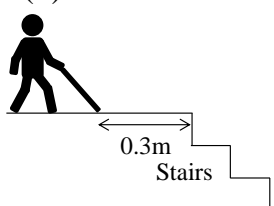

(d) Down-stair

\subsection{Results of experiment}

Fig. 8 shows the verification result by according to the types of obstacles. From the test result, we have found that the real distance is shorter than the estimated distance to a wall, an obstacle, and up-stairs. It is because this system detects the shortest distance to the obstacle by the directivity of the ultrasonic of the distance sensor. As for down-stairs, the real distance is longer than the estimated distance, and so the system detects them within the directivity of the ultrasonic. From the above, we get the difference between the real distance and the estimated distance, and, if the proper threshold $\varepsilon$ is defined, the system can be said to detect obstacles.

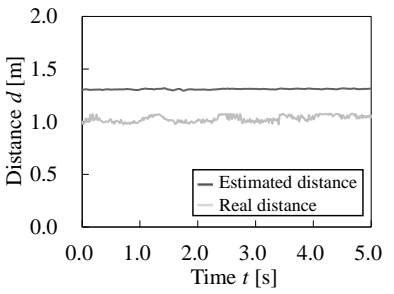

(a) Wall

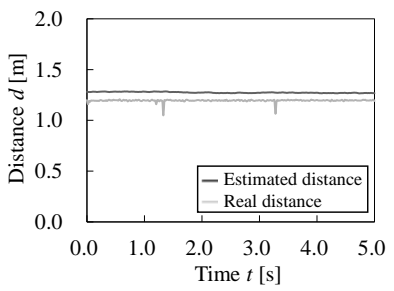

(c) Up-stairs

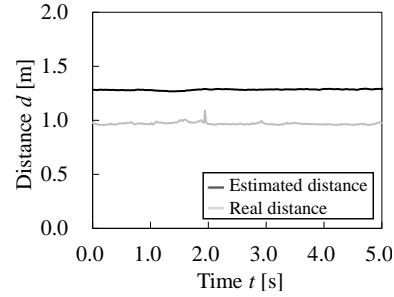

(b) Obstacle

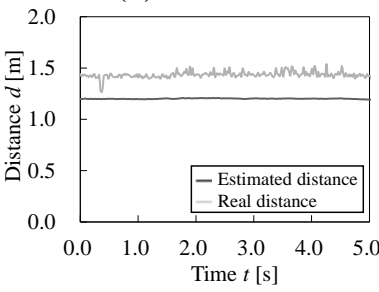

(d) Down-stairs
Fig.8 The verification results

\section{Evaluation experiment supposing walking of visually impaired person}

\subsection{Experiment method on supposition of walking}

Concerning the use of the white walking stick by the visually impaired, there are the slide technique and the touch technique as shown in Fig.9. With the slide technique a walker can detect obstacles by sliding the stick along the ground. With the touch technique a walker can detect obstacles by touching the ground between the shoulders with the end of the stick. In this study, we supposed the walking of a visually impaired person and obstacles are the same as those shown in Fig.7. We did the experiment by the following process.

1. The tester stands $3 \mathrm{~m}$ away from the object of detection.

2. The tester approaches the obstacle waving the stick, and measures the real distance and the estimated distance.

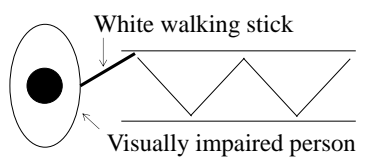

(a) Slide technique

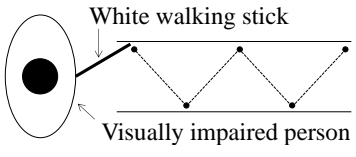

(b) Touch technique
Fig.9 Two ways of using the white walking stick to detect obstacles

\subsection{Experiment result of touch technic}

Fig.10 shows the verification result according to the types of obstacles. In the case of the touch technique with which a visually impaired person walks moving the white walking stick hard, the waves of the real distance and those of the estimated distance were disturbed by the periodical oscillation of the white stick during a walk, making it difficult for us to get the proper difference. 


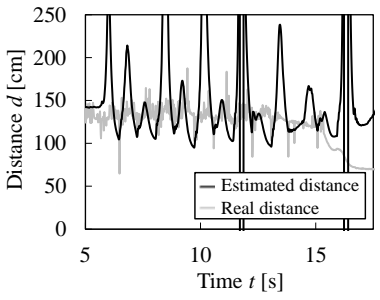

(a) Wall

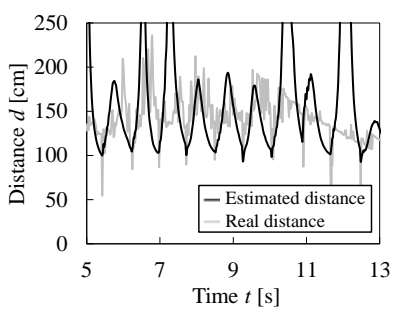

(c)Up-stair

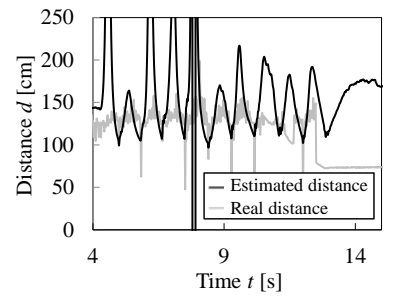

(b) Obstacle

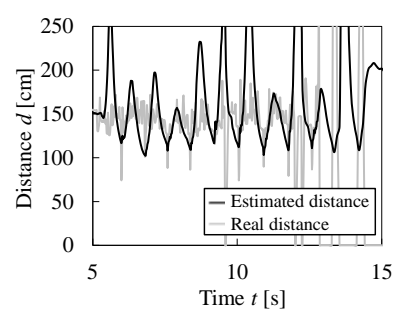

(d) Down-stair
Fig.10 The obstacle detection result at touch technic

\section{Removal of periodical oscillation of white}

\section{walking stick}

\subsection{Method of experiment}

In our study, by using the circuit simulator "LT-spice", we examined the oscillation removal of the measured value. We calculated the frequency component of the estimated distance by FFT before doing the simulation of the oscillation removal.

Fig.11 shows the result of FFT. The Spectrum is rising sharply near $0 \mathrm{~Hz}$. From this result, we thought the estimated distance to form a wave which originally had few rapid changes. So we thought that if we could cut off information other than that near $0 \mathrm{~Hz}$, we would be able to extract the necessary data. To remove the big oscillations, by trial and error, we have used the $\mathrm{RC}$ series low pass filter, as shown in Fig.12, whose resistance $\mathrm{R}$ is $100 \mathrm{k} \Omega$, and whose condenser $\mathrm{C}$ is $200 \mu \mathrm{F}$. As the cutoff frequency is about $0.0008 \mathrm{~Hz}$, we have considered it enough to remove the big oscillations. And we used the condenser $\mathrm{C}$ which is $25 \mu \mathrm{F}$ for real distance data with comparatively small oscillations.

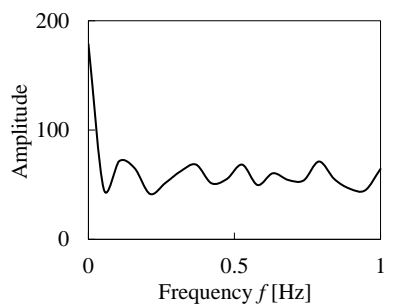

Fig.11 The result of FFT

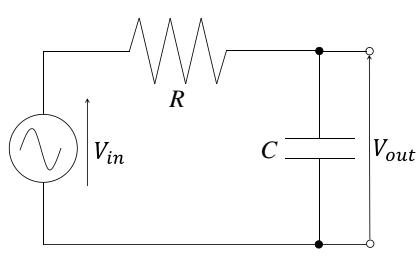

Fig.12 The simulation circuit

\subsection{Result of experiment}

Fig.13 shows the estimated distance and the real distance which were respectively passed through the LPF. In Fig.13 (a), (b) and (d), the oscillations of the estimated distance and the real distance are reduced, and the difference appears clearly. As for the up-stairs in Fig13 (c), the difference before detecting and that after detecting are very similar to each other. After this, by increasing the number of experimental results, we will clarify the problems and improve the system.

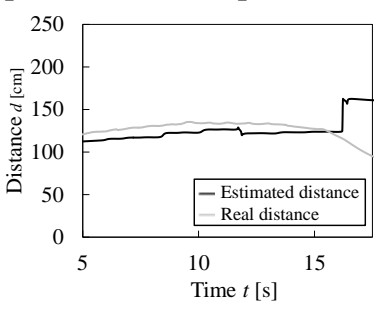

(a) Wall

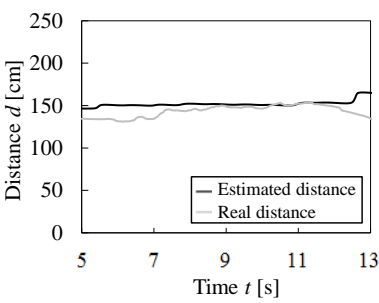

(c) Up-stair

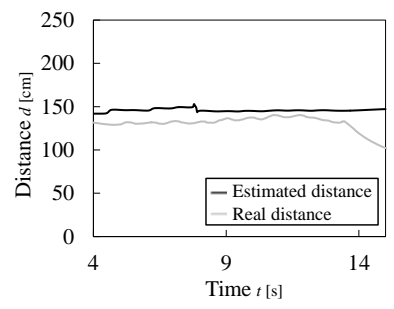

(b) Obstacle

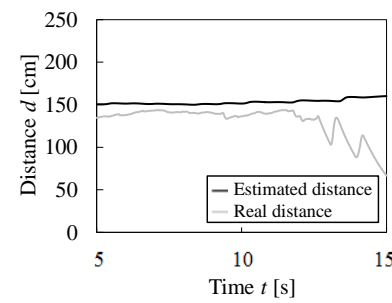

(d) Down-stair
Fig.13 The result of LPF

\section{Conclusion}

In this study, we have built the stationary obstacle detection system which doesn't depend on the height information from the ground to the smartphone. From the result of the test, by using the developed system, we have found it possible to detect obstacles such as the wall, stairs and other obstacles which prevent visually impaired persons from walking. By running a simulation, we have verified that the periodical oscillation from the touch technique used by the visually impaired when walking can be removed by using the LPF. In the future we will install a filter in the circuit and develop the dynamic obstacle detection system using the Doppler sensor.

\section{Acknowledgment}

This work was supported by JSPS KAKENHI Grant Number 15K01492.

\section{Reference}

(1) Y. Tange, S. Takeno and J. Hori, "Development of the Obstacle Detection System Combining Orientation Sensor of Smartphone and Distance Sensor", The 37th Annual International Conference of the IEEE Engineering in Medicine and Biology Society, pp.6696-6699, (2015.8). 